\title{
ASYMPTOTIC NORMALITY IN MIXTURE MODELS
}

\author{
SARA VAN DE GEER
}

ABstRact. We study the estimation of a linear function $\theta_{0}=\int \operatorname{ad} F_{0}$ of a distribution $F_{0}$, using i.i.d. observations of the mixture $p_{F_{0}}=$ $\int k(\cdot, y) d F_{0}(y)$. Let $\hat{F}_{n}$ be the maximum likelihood estimator of $F_{0}$ and $\hat{\theta}_{n}=\int a d \hat{F}_{n}$. We examine the asymptotic distribution of $\hat{\theta}_{n}$. A problem here is that usually, $\hat{F}_{n}$ does not dominate $F_{0}$. Our main aim is to show that this can be overcome by considering the convex combination $\alpha \hat{F}_{n}+(1-\alpha) F_{0}$, with $\alpha<1$.

\section{INTRODUCTION}

Let $X_{1}, \ldots, X_{n}$ be independent identically distributed random variables on $(\mathcal{X}, \mathcal{A})$, with distribution $P$. Suppose that for some $\sigma$-finite measure $\mu$,

$$
p=\frac{d P}{d \mu} \in\left\{p_{F}=\int k(\cdot, y) d F(y): F \in \Lambda\right\},
$$

where $\Lambda$ is the class of all probability measures on a measurable space $(\mathcal{Y}, \mathcal{B})$, and where $k: \mathcal{X} \times \mathcal{Y} \rightarrow[0, \infty)$ is a given kernel with $\int k(x, y) d \mu(x)=1$ for all $y \in \mathcal{Y}$. So

$$
p=p_{F_{0}}=\int k(\cdot, y) d F_{0}(y)
$$

for some $F_{0} \in \Lambda$.

In this paper, we assume that $F_{0}$ is unknown, and that the maximum likelihood estimator $\hat{F}_{n}$ of $F_{0}$ exists. The latter is (not necessarily uniquely) defined by

$$
\sum_{i=1}^{n} \log p_{\hat{F}_{n}}\left(X_{i}\right)=\max _{F \in \Lambda} \sum_{i=1}^{n} \log p_{F}\left(X_{i}\right) .
$$

Consider now functionals of the form

$$
\theta(F)=\int a d F, F \in \Lambda,
$$

with $a$ a given function on $(\mathcal{Y}, \mathcal{B})$. Write $\theta_{0}=\int a d F_{0}$ and $\hat{\theta}_{n}=\int a d \hat{F}_{n}$. We shall investigate the asymptotic behaviour (and efficiency) of the estimator of $\hat{\theta}_{n}$ of $\theta_{0}$. An important issue in this context is the appropriate differentiability of $\theta(F)$. Let us briefly sketch the main idea.

ESAIM: Probability and Statistics is an electronic journal with URL address http://www.emath.fr/ps.

Received by the journal 8 March 1995. Revised 2 June 1995. Accepted for publication 28 September 1995 
Consider a pair of random variables $(X, Y)$ with values in $\mathcal{X} \times \mathcal{Y}$, let $k(\cdot, y)$ be the density of $X$ given $Y=y$, and $F_{0}$ the distribution of $Y$. For a function $b: \mathcal{X} \rightarrow \mathbf{R}$, write

$$
E(b(X) \mid Y=y)=A^{*} b(y) .
$$

Thus

$$
A^{*} b(y)=\int k(x, y) b(x) d \mu(x) .
$$

Write for $h: \mathcal{Y} \rightarrow \mathbf{R}$,

$$
E(h(Y) \mid X=x)=A_{F_{0}} h(x)
$$

where

$$
A_{F} h(x)=\frac{\int k(x, y) h(y) d F(y)}{p_{F}(x)}
$$

If for some $b$,

$$
E(b(X) \mid Y=y)=a(y), \text { for } F_{0}-\text { almost all } y,
$$

then clearly

$$
E(b(X))=\theta_{0} .
$$

So if (1.1) holds for some $b \in L_{2}(P)$ not depending on $F_{0}$, then $(1 / n) \sum_{i=1}^{n} b\left(X_{i}\right)$ is a $\sqrt{n}$-consistent and asymptotically normal estimator of $\theta_{0}$. We say that $\theta\left(F_{0}\right)$ is differentiable at $F_{0}$ if a solution of (1.1) exists. Note that if $k(\cdot, y)$ is a complete family for $y$ in the support of $F_{0}$, there is at most one solution of (1.1). In general, there may also be several solutions, in which case we would like to take the one with the smallest variance. But such a solution possibly depends on $F_{0}$. The arguments below indicate that perhaps the maximum likelihood procedure automatically picks the best solution, with the estimator $\hat{F}_{n}$ plugged in for $F_{0}$.

We shall now discuss the solution with the smallest variance. First, we center the functions. Instead of $a$ in (1.1), we consider the gradient of $\theta(F)$, which is defined as

$$
\psi_{F}=a-\int a d F
$$

If for some $h_{F} \in L_{\infty}(F)$ with $\int h_{F} d F=0$,

$$
A^{*} A_{F} h_{F}=\psi_{F}, F-\text { a.s., }
$$

we call

$$
b_{F}=A_{F} h_{F}
$$

the efficient influence curve at $\theta(F)$. Note that $b_{F}$ is also centered now: $\int b_{F} d P_{F}=0$. It follows from Van der Vaart (1991) that $(1 / n) \int b_{F_{0}}^{2} d P$ is a lower bound for the asymptotic variance of an estimator of $\theta_{0}$. He considers parametric submodels with Hilbert space structure to arrive at results of this type in a very general context. In our case, the parametric submodel would 
be indexed by $\Lambda_{F}=\left\{F_{t}:\left(d F_{t}\right)^{1 / 2}=\left(1+\frac{1}{2} t h_{F}\right)(d F)^{1 / 2},|t|\right.$ small $\}$. For this reason, we call $h_{F}$ a direction (along which one can consider a submodel). The assumptions $h_{F} \in L_{\infty}(F)$ and $\int h_{F} d F=0$ ensure that indeed $\Lambda_{F} \subset \Lambda$.

Suppose now that the efficient influence curves $b_{\hat{F}_{n}}$ and $b_{F_{0}}$ exist. So $b_{\hat{F}_{n}}=A_{\hat{F}_{n}} h_{\hat{F}_{n}}$ for some $h_{\hat{F}_{n}} \in L_{\infty}\left(\hat{F}_{n}\right)$, and

$$
A^{*} b_{\hat{F}_{n}}=\psi_{\hat{F}_{n}}, \hat{F}_{n}-\text { a.s.. }
$$

Observe that $\hat{F}_{n}$ is an interior point of $\left\{\hat{F}_{n, t}: d \hat{F}_{n, t}=\left(1+t h_{\hat{F}_{n}}\right) d \hat{F}_{n},|t|\right.$ small $\}$. So we have

$$
\left.\frac{d}{d t} \sum_{i=1}^{n} \log p_{\hat{F}_{n, t}}\left(X_{i}\right)\right|_{t=0}=0
$$

or

$$
\sum_{i=1}^{n} b_{\hat{F}_{n}}\left(X_{i}\right)=0 .
$$

Write this as

$$
\int b_{\hat{F}_{n}} d P_{n}=0
$$

with $P_{n}$ the empirical distribution based on $X_{1}, \ldots, X_{n}$ (see (2.1)). Now, let us compare this with $\int b_{\hat{F}_{n}} d P$. Changing the order of integration gives

$$
\int b_{\hat{F}_{n}} d P=\int A^{*} b_{\hat{F}_{n}} d F_{0}
$$

Moreover, if $\hat{F}_{n}$ dominates $F_{0}$, then (1.4) yields

$$
\int A^{*} b_{\hat{F}_{n}} d F_{0}=\int \psi_{\hat{F}_{n}} d F_{0}=\theta_{0}-\hat{\theta}_{n}
$$

So then we have the identity of van der Laan $(1993,1994)$ :

$$
\hat{\theta}_{n}-\theta_{0}=\int b_{\hat{F}_{n}} d\left(P_{n}-P\right) .
$$

Finally, if $b_{\hat{F}_{n}}$ converges to $b_{F_{0}}$ in an appropriate sense, one obtains asymptotic normality (and efficiency) of $\hat{\theta}_{n}$.

The problem is now that the assumption that $\hat{F}_{n}$ dominates $F_{0}$ is often not valid. Nevertheless, van der Laan (1993) presents some examples that show that the identity (1.5) can hold even when $\hat{F}_{n}$ does not dominate $F_{0}$. We shall however be concerned with the situation where the identity (1.5) is not necessarily true.

Our approach is to consider for each $0 \leq \alpha<1$ and $F \in \Lambda$, the convex combination

$$
F_{\alpha}=\alpha F+(1-\alpha) F_{0} .
$$

Then indeed, $\hat{F}_{n, \alpha}$ dominates $F_{0}$. We shall obtain asymptotic normality of $\hat{\theta}_{n}$ by choosing $\alpha=\hat{\alpha}_{n}$ in such a way that it tends to one at an appropriate speed. 
The paper is organized as follows. In Section 2, we derive a linear approximation for $\hat{\theta}_{n}$. Asymptotic normality and efficiency follow from this. The conditions we need to arrive at the result are consistency of the maximum likelihood estimator (obtained by separate means), differentiability of $\theta(F)$ at $F=F_{\alpha}, 0 \leq \alpha<1$, bounded directions and suitable continuity conditions on the influence curves. A discussion of these conditions can be found in Section 3. Section 4 presents some examples.

\section{ASYMPTOTIC NORMALITY}

As pseudo-metric on $\Lambda$, we take the Hellinger distance between the mixtures:

$$
d(F, \tilde{F})=h\left(p_{F}, p_{\tilde{F}}\right)=\left(\frac{1}{2} \int\left(\sqrt{p_{F}}-\sqrt{p_{\tilde{F}}}\right)^{2} d \mu\right)^{1 / 2}, F, \tilde{F} \in \Lambda .
$$

Consistency of $\hat{F}_{n}$ in this metric holds in fairly general situations. It is closely related to the further assumptions as stated in Condition 1 (see Section 3.1 for more details).

We use the notation

$$
P_{n}=\frac{1}{n} \sum_{i=1}^{n} \delta_{X_{i}}
$$

i.e. $P_{n}$ is the empirical distribution that puts mass $(1 / n)$ at each of the observations $X_{1}, \ldots, X_{n}$. Moreover, we shall make frequent use of stochastic order symbols. If $\left\{Z_{n}\right\}$ is a sequence of real-valued random variables, and $\left\{k_{n}\right\}$ a sequence of positive numbers, then we say that $Z_{n}=O_{\mathbf{P}}\left(k_{n}\right)$ if

$$
\lim _{M \rightarrow \infty} \limsup _{n \rightarrow \infty} \mathbf{P}\left(\left|Z_{n}\right|>k_{n} M\right)=0 .
$$

Similarily, $Z_{n}=o_{\mathbf{P}}\left(k_{n}\right)$ means that for all $\epsilon>0$,

$$
\lim _{n \rightarrow \infty} \mathbf{P}\left(\left|Z_{n}\right|>k_{n} \epsilon\right)=0
$$

Condition 1. (consistency and rates). The estimators $\hat{F}_{n}$ and $\hat{\theta}_{n}$ are consistent, i.e.

$$
d\left(\hat{F}_{n}, F_{0}\right)=o_{\mathbf{P}}(1)
$$

and

$$
\left|\hat{\theta}_{n}-\theta_{0}\right|=o_{\mathbf{P}}(1) .
$$

Moreover, for some $\delta_{n}^{2}=o\left(n^{-1 / 2}\right)$,

$$
\int \log \left(\frac{2 p_{\hat{F}_{n}}}{p_{\hat{F}_{n}}+p_{F_{0}}}\right) d P_{n}=O_{\mathbf{P}}\left(\delta_{n}^{2}\right) .
$$

Condition 2 (differentiability in a neighbourhood of $F_{0}$ and existence of efficient influence curves). For some $\epsilon>0$, and for all $0 \leq \alpha<1$ and $F \in \Lambda$ with $d\left(F, F_{0}\right) \leq \epsilon$, we have

$$
A^{*} b_{F_{\alpha}}=\psi_{F_{\alpha}}, F_{\alpha}-\text { a.s. }
$$


for $b_{F_{\alpha}}$ satisfying

$$
b_{F_{\alpha}}(x)=A_{F_{\alpha}} h_{F_{\alpha}}(x), p_{F_{\alpha}}(x)>0,
$$

for some $h_{F_{\alpha}} \in L_{\infty}\left(F_{\alpha}\right)$, with $\int h_{F_{\alpha}} d F_{\alpha}=0$.

Condition 3. (control on the direction $h_{F_{\alpha}}$ ). For some $\epsilon>0$ and $M<\infty$,

$$
\sup _{d\left(F, F_{0}\right) \leq \epsilon} \sup _{0 \leq \alpha<1} \sup _{y \in \operatorname{support}\left(F_{\alpha}\right)}\left|(1-\alpha) h_{F_{\alpha}}(y)\right| \leq M .
$$

CONDITION 4. (control on the efficient influence curves $b_{F_{\alpha}}$ ). The information for estimating $\theta_{0}$ is positive:

$$
\int b_{F_{0}}^{2} d P>0
$$

Moreover, the influence curves are uniformly bounded: for some $\epsilon>0$,

$$
\sup _{d\left(F, F_{0}\right) \leq \epsilon} \sup _{0 \leq \alpha<1} \sup _{p_{F_{\alpha}}(x)>0}\left|b_{F_{\alpha}}(x)\right|<\infty .
$$

Finally, for $0<\epsilon_{n} \rightarrow 0$,

$$
\sup _{d\left(F, F_{0}\right) \leq \epsilon_{n}} \sup _{0 \leq \alpha<1} \int b_{F_{\alpha}}^{2} d P_{n}=\int b_{F_{0}}^{2} d P+o_{\mathbf{P}}(1),
$$

and

$$
\sup _{d\left(F, F_{0}\right) \leq \epsilon_{n}} \sup _{0 \leq \alpha<1} \int\left(b_{F_{\alpha}}-b_{F_{0}}\right) d\left(P_{n}-P\right)=o_{\mathbf{P}}\left(n^{-1 / 2}\right) .
$$

Theorem 2.1. Assume that conditions 1-4 are met. Then

$$
\hat{\theta}_{n}-\theta_{0}=\int b_{F_{0}} d\left(P_{n}-P\right)+o_{\mathbf{P}}\left(n^{-1 / 2}\right) .
$$

Proof. By (2.4), we can find a non-random increasing function $\gamma:(0, \infty) \rightarrow$ $(0, \infty)$, satisfying

$$
\begin{gathered}
\gamma(x) \rightarrow 0 \text { for } x \rightarrow 0, \\
\frac{x}{\gamma(x)} \leq \frac{1}{2} \text { for all } x>0,
\end{gathered}
$$

and

$$
\frac{x}{\gamma(x)} \rightarrow 0 \text { for } x \rightarrow 0
$$

such that $\delta_{n}^{2}=o\left(n^{-1 / 2}\right) \gamma\left(n^{-1 / 2}\right)$. This gives

$$
\int \log \left(\frac{2 p_{\hat{F}_{n}}}{p_{\hat{F}_{n}}+p_{F_{0}}}\right) d P_{n}=o_{\mathbf{P}}\left(n^{-1 / 2}\right) \gamma\left(n^{-1 / 2}\right) .
$$


Choose

$$
1-\hat{\alpha}_{n}=\frac{\left|\hat{\theta}_{n}-\theta_{0}\right|+n^{-1 / 2}}{\gamma\left(\left|\hat{\theta}_{n}-\theta_{0}\right|+n^{-1 / 2}\right)} .
$$

Then $\hat{\alpha}_{n}<1$ and by (2.14), $\hat{\alpha}_{n} \geq 1 / 2$. Since $\left|\hat{\theta}_{n}-\theta_{0}\right|=o_{\mathbf{P}}(1),(2.15)$ implies that $\left(1-\hat{\alpha}_{n}\right)=o_{\mathbf{P}}(1)$. Furthermore, $(2.13)$ yields

$$
\frac{\left|\hat{\theta}_{n}-\theta_{0}\right|}{1-\hat{\alpha}_{n}}=o_{\mathbf{P}}(1)
$$

as well as

$$
\frac{n^{-1 / 2}}{1-\hat{\alpha}_{n}}=o_{\mathbf{P}}(1)
$$

Define

$$
\tilde{F}_{n}=\hat{\alpha}_{n} \hat{F}_{n}+\left(1-\hat{\alpha}_{n}\right) F_{0} .
$$

Let $\epsilon>0$ be small enough, so that (2.5), (2.6), (2.7) and (2.9) in conditions $2-4$ are fulfilled for this value of $\epsilon$, and let $D_{n}$ be the set

$$
D_{n}=\left\{d\left(\hat{F}_{n}, F_{0}\right) \leq \epsilon\right\} .
$$

Because $\hat{\alpha}_{n}<1$, we can find on $D_{n}$ an influence curve $b_{\tilde{F}_{n}}$ and a direction $h_{\tilde{F}_{n}}$, such that

$$
A^{*} b_{\tilde{F}_{n}}=\psi_{\tilde{F}_{n}}
$$

and

$$
b_{\tilde{F}_{n}}(x)=A_{\tilde{F}_{n}} h_{\tilde{F}_{n}}(x), x \in\left\{p_{\tilde{F}_{n}}>0\right\} .
$$

We can write

$$
\int b_{\tilde{F}_{n}} d P_{n} 1\left\{D_{n}\right\}=\int b_{\tilde{F}_{n}} d\left(P_{n}-P\right) 1\left\{D_{n}\right\}+\int b_{\tilde{F}_{n}} d P 1\left\{D_{n}\right\} .
$$

Because $d\left(\hat{F}_{n}, F_{0}\right)=o_{\mathbf{P}}(1)$, we have that $1\left\{D_{n}\right\}=1+o_{\mathbf{P}}(1)$. So, using (2.11),

$$
\int b_{\tilde{F}_{n}} d\left(P_{n}-P\right) 1\left\{D_{n}\right\}=\int b_{F_{0}} d\left(P_{n}-P\right)+o_{\mathbf{P}}\left(n^{-1 / 2}\right)=O_{\mathbf{P}}\left(n^{-1 / 2}\right)
$$

Since $\tilde{F}_{n}$ dominates $F_{0}$, and $\hat{\alpha}_{n}=1+o_{\mathbf{P}}(1)$,

$$
\int b_{\tilde{F}_{n}} d P 1\left\{D_{n}\right\}=-\hat{\alpha}_{n}\left(\hat{\theta}_{n}-\theta_{0}\right) 1\left\{D_{n}\right\}=-\left(1+o_{\mathbf{P}}(1)\right)\left(\hat{\theta}_{n}-\theta_{0}\right) .
$$

Insert (2.22) and (2.23) into (2.21) to get that

$$
\int b_{\tilde{F}_{n}} d P_{n} 1\left\{D_{n}\right\}=\int b_{F_{0}} d\left(P_{n}-P\right)+o_{\mathbf{P}}\left(n^{-1 / 2}\right)-\left(1+o_{\mathbf{P}}(1)\right)\left(\hat{\theta}_{n}-\theta_{0}\right)
$$

Let

$$
\hat{t}_{n}=\frac{\int b_{\tilde{F}_{n}} d P_{n}}{\left(1-\hat{\alpha}_{n}\right) \int b_{F_{0}}^{2} d P} 1\left\{D_{n}\right\} .
$$


Equality (2.24), together with (2.8), (2.18) and (2.19) imply that

$$
\hat{t}_{n}=o_{\mathbf{P}}(1) \text {. }
$$

Take $M$ as in (2.7) and $E_{n}=D_{n} \cap\left\{\left|\hat{t}_{n}\right|<1 / M\right\}$. Define on $\left\{E_{n}\right\}$,

$$
d \tilde{F}_{n}\left(\hat{t}_{n}\right)=\left(1+\hat{t}_{n}\left(1-\hat{\alpha}_{n}\right) h_{\tilde{F}_{n}}\right) d \tilde{F}_{n} .
$$

Then on $\left\{E_{n}\right\}$,

$$
\tilde{F}_{n}\left(\hat{t}_{n}\right) \in \Lambda \text {. }
$$

Moreover, from (2.26) we know that $1\left\{E_{n}\right\}=1+o_{\mathbf{P}}(1)$, so that by $(2.9)$ and $(2.10)$,

$$
\begin{gathered}
\int \log \left(\frac{p_{\tilde{F}_{n}\left(\hat{t}_{n}\right)}}{p_{\tilde{F}_{n}}}\right) d P_{n} 1\left\{E_{n}\right\}=\int \log \left(1+\hat{t}_{n}\left(1-\hat{\alpha}_{n}\right) b_{\tilde{F}_{n}}\right) d P_{n} 1\left\{E_{n}\right\} \\
=\hat{t}_{n}\left(1-\hat{\alpha}_{n}\right) \int b_{\tilde{F}_{n}} d P_{n} 1\left\{E_{n}\right\}-\frac{1}{2} \hat{t}_{n}^{2}\left(1-\hat{\alpha}_{n}\right)^{2} \int b_{\tilde{F}_{n}}^{2} d P_{n} 1\left\{E_{n}\right\}\left(1+o_{\mathbf{P}}(1)\right) \\
=\frac{1}{2} \frac{\left(\int b_{\tilde{F}_{n}} d P_{n}\right)^{2}}{\int b_{F_{0}}^{2} d P} 1\left\{E_{n}\right\}\left(1+o_{\mathbf{P}}(1)\right) .
\end{gathered}
$$

Since $\hat{F}_{n}$ maximizes the likelihood, and $(2.27)$ holds on $E_{n}$, we have

$$
\int \log p_{\hat{F}_{n}} d P_{n} 1\left\{E_{n}\right\} \geq \int \log p_{\tilde{F}_{n}\left(\hat{t}_{n}\right)} d P_{n} 1\left\{E_{n}\right\} .
$$

Furthermore, the concavity of the log-function and the fact that $\hat{\alpha}_{n} \geq 1 / 2$ yield

$\int \log p_{\tilde{F}_{n}} d P_{n} \geq\left(2 \hat{\alpha}_{n}-1\right) \int \log p_{\hat{F}_{n}} d P_{n}+2\left(1-\hat{\alpha}_{n}\right) \int \log \left(\frac{p_{\hat{F}_{n}}+p_{F_{0}}}{2}\right) d P_{n}$.

Combine $(2.28),(2.29)$ and $(2.30)$ to find

$$
2\left(1-\hat{\alpha}_{n}\right) \int \log \left(\frac{2 \hat{p}_{\hat{F}_{n}}}{p_{\hat{F}_{n}}+p_{F_{0}}}\right) d P_{n} \geq \frac{1}{2} \frac{\left(\int b_{\tilde{F}_{n}} d P_{n}\right)^{2}}{\int b_{F_{0}}^{2} d P} 1\left\{E_{n}\right\}\left(1+o_{\mathbf{P}}(1)\right) .
$$

From (2.16) and (2.17), we know that the left-hand side of this equality is

$$
\begin{gathered}
\left(1-\hat{\alpha}_{n}\right) o_{\mathbf{P}}\left(n^{-1 / 2}\right) \gamma\left(n^{-1 / 2}\right)=\left(\left|\hat{\theta}-\theta_{0}\right|+n^{-1 / 2}\right) \frac{\gamma\left(n^{-1 / 2}\right)}{\gamma\left(\left|\hat{\theta}_{n}-\theta_{0}\right|+n^{-1 / 2}\right)} o_{\mathbf{P}}\left(n^{-1 / 2}\right) \\
=\left(\left|\hat{\theta}_{n}-\theta_{0}\right|+n^{-1 / 2}\right) o_{\mathbf{P}}\left(n^{-1 / 2}\right),
\end{gathered}
$$

where in the last step, we used that $\gamma$ is increasing. In view of (2.24), the right-hand side of (2.31) is of the form

$$
\left(O_{\mathbf{P}}\left(n^{-1 / 2}\right)-\left(1+o_{\mathbf{P}}(1)\right)\left(\hat{\theta}_{n}-\theta_{0}\right)\right)^{2}\left(\frac{1+o_{\mathbf{P}}(1)}{\int b_{F_{0}}^{2} d P}\right) .
$$


So we find from (2.31) that

$$
\left|\hat{\theta}_{n}-\theta_{0}\right|^{2} \leq \max \left\{O_{\mathbf{P}}\left(n^{-1}\right),\left(\left|\hat{\theta}_{n}-\theta_{0}\right|+n^{-1 / 2}\right) o_{\mathbf{P}}\left(n^{-1 / 2}\right)\right\}
$$

which implies $\left|\hat{\theta}_{n}-\theta_{0}\right|=O_{\mathbf{P}}\left(n^{-1 / 2}\right)$. But then, the left-hand side of (2.31) is $o_{\mathbf{P}}\left(n^{-1}\right)$, so that it reads

$$
\left(\int b_{F_{0}} d\left(P_{n}-P\right)+o_{\mathbf{P}}\left(n^{-1 / 2}\right)-\left(1+o_{\mathbf{P}}(1)\right)\left(\hat{\theta}_{n}-\theta_{0}\right)\right)^{2}\left(1+o_{\mathbf{P}}(1)\right)=o_{\mathbf{P}}\left(n^{-1}\right)
$$

In other words,

$$
\hat{\theta}_{n}-\theta_{0}=\int b_{F_{0}} d\left(P_{n}-P\right)+o_{\mathbf{P}}\left(n^{-1 / 2}\right) .
$$

\section{COMMENTS ON CONDITIONS 1-4}

Conditions 1 and 4 can be verified using the concept of entropy. Therefore, we introduce the following definitions. Let $Q$ be a probability measure on $(\mathcal{X}, \mathcal{A})$ and $\mathcal{G} \subset \mathcal{L}_{q}(Q), q \geq 1$.

Definition 1 The $\delta$-covering number $N_{q}(\delta, \mathcal{G}, Q)$ of $\mathcal{G}$ is defined as the number of balls with radius $\delta$ necessary to cover $\mathcal{G}$. More precisely, let $\left\{g_{j}\right\}_{j=1}^{m}$ be such that for each $g \in \mathcal{G}$ there is a $j \in\{1, \ldots, m\}$ such that

$$
\int\left(g-g_{j}\right)^{q} d Q \leq \delta^{q}
$$

Then $N_{q}(\delta, \mathcal{G}, Q)$ is the smallest $m$ for which such a collection $\left\{g_{j}\right\}_{j=1}^{m}$ exists. The $\delta$-entropy of $\mathcal{G}$ is $H_{q}(\delta, \mathcal{G}, Q)=\log N_{q}(\delta, \mathcal{G}, Q) \vee 1$.

Definition 2. Let $\left\{\left[g_{j}^{L}, g_{j}^{U}\right]\right\}_{j=1}^{m}$ be such that for all $g \in \mathcal{G}$ there is a $j \in\{1 \ldots, m\}$ such that

$$
g_{j}^{L} \leq g \leq g_{j}^{U}
$$

and

$$
\int\left(g_{j}^{U}-g_{j}^{L}\right)^{q} d Q \leq \delta^{q}
$$

Write $N_{q}^{B}(\delta, \mathcal{G}, Q)$ for the smallest $m$ for which such a collection $\left\{\left[g_{j}^{L}, g_{j}^{U}\right]\right\}_{j=1}^{m}$ exists. Then $H_{q}^{B}(\delta, \mathcal{G}, Q)=\log N_{q}^{B}(\delta, \mathcal{G}, Q) \vee 1$ is called the $\delta$-entropy with bracketing.

\subsection{ON CONDITION 1}

Suppose that $\mathcal{Y}$ is a locally compact Hausdorff space with countable base, and that $\mathcal{B}$ is the Borel $\sigma$-algebra. Let $\mathcal{C}_{0}$ be the class of all functions $c: \mathcal{Y} \rightarrow \mathbf{R}$ that vanish at infinity. If $k(x, \cdot) \in \mathcal{C}_{0}$ for $\mu$-almost all $x \in \mathcal{X}$, then $d\left(\hat{F}_{n}, F_{0}\right) \rightarrow 0$ almost surely (see Pfanzagl (1988)). 
Denote the class of all measures $F$ on $(\mathcal{Y}, \mathcal{B})$ with $F(\mathcal{Y}) \leq 1$ by $\Lambda^{*}$. The vague topology on $\Lambda^{*}$ is the smallest topology such that $F \mapsto \int c d F$ is continuous for every $c \in \mathcal{C}_{0}$. Let $\tau$ be the metric corresponding to the vague topology. We say that $F_{0}$ is identifiable (for the metric $\tau$ ) if for all $F \in \Lambda^{*}$, $d\left(F, F_{0}\right)=0$ implies $\tau\left(F, F_{0}\right)=0$. If $F_{0}$ is identifiable, then $d\left(\hat{F}_{n}, F_{0}\right) \rightarrow 0$ almost surely implies $\tau\left(\hat{F}_{n}, F_{0}\right) \rightarrow 0$ almost surely. So in particular, then $\left|\hat{\theta}_{n}-\theta_{0}\right| \rightarrow 0$ almost surely, whenever $a \in \mathcal{C}_{0}$. More details can be found in e.g. Pfanzagl (1988) or Van de Geer (1993a).

Let us now investigate the rate of convergence for the log-likelihood ratio. Note first of all that

$$
0 \leq \int \log \left(\frac{2 p_{\hat{F}_{n}}}{p_{\hat{F}_{n}}+p_{F_{0}}}\right) d P_{n} \leq \frac{1}{2} \int \log \left(\frac{p_{\hat{F}_{n}}}{p_{F_{0}}}\right) d P_{n}
$$

so that nothing is lost (and indeed something could be gained) by comparing $p_{\hat{F}_{n}}$ with the convex combination $\left(p_{\hat{F}_{n}}+p_{F_{0}}\right) / 2$ instead of with $p_{F_{0}}$.

Lemma 3.1. Suppose that

$$
\int_{0}^{1} \sqrt{H_{2}^{B}(\delta, \mathcal{G}, P)} d \delta<\infty
$$

where

$$
\mathcal{G}=\left\{\sqrt{\frac{p_{F}+p_{F_{0}}}{p_{F_{0}}}}: F \in \Lambda\right\} .
$$

Then

$$
\int \log \left(\frac{p_{\hat{F}_{n}}}{p_{F_{0}}}\right) d P_{n}=O_{\mathbf{P}}\left(\delta_{n}^{2}\right), \text { with } \delta_{n}^{2}=o\left(n^{-1 / 2}\right) .
$$

Proof. See Van de Geer (1995). A slight modification can be found in Wong and Shen (1992).

We call the left-hand side of (3.4) the entropy integral. If the entropy integral diverges, suboptimal rates can emerge (see Birgé and Massart (1993)).

Lemma 3.2 below makes use of the special structure of the mixing model. We need the following notation: for $\sigma \geq 0$,

$$
\tau_{1}^{2}(\sigma)=\int_{p_{F_{0}} \leq \sigma} p_{F_{0}} d \mu
$$

and

$$
\tau_{2}^{2}(\sigma)=\int_{p_{F_{0}}>\sigma} \frac{1}{p_{F_{0}}} d \mu
$$

Lemma 3.2. Let $\mathcal{K}=\{k(\cdot, y): y \in \mathcal{Y}\}$. Suppose that the functions in $\mathcal{K}$ are uniformly bounded, and that for all probability measures $Q$ with finite support,

$$
N_{2}(\delta, \mathcal{K}, Q) \leq A \delta^{-w}, \delta>0
$$


where the constants $A$ and $w$ do not depend on $Q$. Let $0 \leq \sigma_{n} \rightarrow 0$, $\delta_{n} \geq \tau_{1}\left(\sigma_{n}\right) \vee n^{-(2+w) /(4+4 w)}\left(\tau_{2}\left(\sigma_{n}\right)\right)^{w /(2+2 w)}$. Then

$$
\int \log \left(\frac{2 p_{\hat{F}_{n}}}{p_{\hat{F}_{n}}+p_{F_{0}}}\right) d P_{n}=O_{\mathbf{P}}\left(\delta_{n}^{2}\right) .
$$

Proof See Van de Geer (1993b).

Lemma 3.2 may not yield the optimal rate, but all we need here is a rate faster than $n^{-1 / 2}$. This is the case if $\tau_{2}\left(\sigma_{n}\right)=o\left(n^{\frac{1}{2 w}}\right)$. To put it differently, if we define

$$
\tau_{1}^{-1}(\delta)=\sup \left\{\sigma: \tau_{1}(\sigma) \leq \delta^{2}\right\},
$$

then $\delta_{n}^{2}=o\left(n^{-1 / 2}\right)$ if

$$
\tau_{2}\left(\tau_{1}^{-1}(\delta)\right)=o\left(\delta^{-\frac{2}{w}}\right) \text { for } \delta \downarrow 0 .
$$

\subsection{ON CONDITION 2}

It is natural to require (2.5) and (2.6) for $\alpha=0$. The fact that we need these equalities for all $0 \leq \alpha<1$ is closely related to being able to estimate the efficient influence curve. However, it should be noted that we only assume $b_{F}$ to exist for certain $F$ that dominate $F_{0}$.

In some applications, $b_{\hat{F}_{n}}$ does exist, but this usually will not help to simplify the proofs (see Section 4 for an example).

\subsection{ON CONDITION 3}

Here, we assume that $h_{F_{\alpha}}$ behaves like $1 /(1-\alpha)$. Clearly, this allows misbehaviour for $\alpha \rightarrow 1$, but it also requires $h_{F_{\alpha}}$ to be bounded. In some applications, this reduces to assuming that $h_{F_{0}}$ is bounded. The proof of Theorem 2.1 reveals that we need that for all $t$ sufficiently small, $d F_{\alpha}(t) / d F_{\alpha}=1+t(1-\alpha) h_{F_{\alpha}}$ exists and is non-negative, i.e., that $F_{\alpha}(t) \in \Lambda$. If for some function $g, d g / d F_{0}$ exists and is bounded, say by $C$, then also $d g / d F_{\alpha}$ exists and $(1-\alpha) d g / d F_{\alpha}$ is bounded by $C$. This is the reason why in Example 4.1 and 4.3 our approach works. But it fails in Example 4.2!

\subsection{On Condition 4}

Let us present a brief overview of some results from empirical process theory, that can be applied in this context. We cite them from Pollard (1984) and Ossiander (1987). Throughout, we assume that the necessary measurability conditions are satisfied.

Consider a class $\mathcal{G}$ of functions on $(\mathcal{X}, \mathcal{A})$, with envelope

$$
G=\sup _{g \in \mathcal{G}}|g| .
$$

The class $\mathcal{G}$ is called a Glivenko-Cantelli class if

$$
\sup _{g \in \mathcal{G}}\left|\int g d\left(P_{n}-P\right)\right| \rightarrow 0, \text { almost surely. }
$$


It is called a Donsker class if $\sqrt{n} \int g d\left(P_{n}-P\right)$ converges in distribution to a mean zero Gaussian process on $\mathcal{G}$. This limiting process is assumed to have continuous sample paths with respect to $\rho(\cdot, \cdot)$, with

$$
\rho\left(g_{1}, g_{2}\right)^{2}=\int\left(g_{1}-g_{2}\right)^{2} d P-\left(\int\left(g_{1}-g_{2}\right) d P\right)^{2} .
$$

Necessary and sufficient conditions for $\mathcal{G}$ to be a Gliven ko-Cantelli class are:

$$
G \in L_{1}(P)
$$

and

$$
H_{1}\left(\delta, \mathcal{G}, P_{n}\right)=o_{\mathbf{P}}(n), \delta>0 .
$$

If $\mathcal{G}$ is a Donsker class, then the asymptotic equicontinuity condition holds, i.e. for all $\epsilon>0$ there exists a $\delta>0$ such that

$$
\limsup _{n \rightarrow \infty} \mathbf{P}\left(\sup _{g \in \mathcal{G}_{\delta}}\left|\sqrt{n} \int g_{\delta} d\left(P_{n}-P\right)\right|>\epsilon\right)<\epsilon,
$$

with $\mathcal{G}_{\delta}=\left\{\left(g_{1}-g_{2}\right): g_{1}, g_{2} \in \mathcal{G}, \rho\left(g_{1}, g_{2}\right) \leq \delta\right\}$. Sufficient conditions for $\mathcal{G}$ to be a Donsker class are:

$$
G \in L_{2}(P)
$$

and

$$
\int_{0}^{1} \sqrt{H_{2}^{B}(\delta, \mathcal{G}, P)} d \delta<\infty
$$

Condition (3.8) may be replaced by

$$
\int_{0}^{1} \sqrt{\lambda(\delta, \mathcal{G})} d \delta<\infty
$$

where

$$
\lambda(\delta, \mathcal{G}) \geq \sup _{Q} H_{2}\left(\delta\left(\int G^{2} d Q\right)^{1 / 2}, \mathcal{G}, Q\right)
$$

and where the supremum is taken over all probability measures $Q$ with finite support.

Suppose now that $(2.9)$ is met, and that for $0<\epsilon_{n} \rightarrow 0$,

$$
\sup _{d\left(F, F_{0}\right) \leq \epsilon_{n}} \sup _{0 \leq \alpha<1} \int\left(b_{F_{\alpha}}-b_{F_{0}}\right)^{2} d P \rightarrow 0 .
$$

Then it is clear from the above that (2.10) and (2.11) are fulfilled if one of the entropy conditions (3.8) or (3.9) holds, with

$$
\mathcal{G}=\left\{b_{F_{\alpha}}: d\left(F, F_{0}\right) \leq \epsilon, 0 \leq \alpha<1\right\} .
$$

In many applications however, no explicit expressions are available for the efficient influence curves, so that it may still be difficult to check the entropy conditions. 


\section{EXAMPLES}

EXample 4.1: interval Censored obervations, Case I

Suppose one observes $X_{i}=\left(T_{i}, \beta_{i}\right)$, where $\beta_{i}=1\left\{Y_{i} \leq T_{i}\right\}, Y_{i}$ and $T_{i}$ are independent random variables, both with values in a bounded interval, say $(0,1]$, and where $T_{i}$ has (unknown) distribution $G, i=1, \ldots, n$. This is one of the models studied in Groeneboom and Wellner (1992). The density of $X_{i}$ with respect to $G \times \nu, \nu$ being the counting measure on $\{0,1\}$, is

$$
p_{F_{0}}(t, \beta)=\beta F_{0}(t)+(1-\beta)\left(1-F_{0}(t)\right)=\int k(t, \beta, y) d F_{0}(y),
$$

with $k(t, \beta, y)=\beta 1\{y \leq t\}+(1-\beta) 1\{y>t\}$.

Lemma 4.1. Suppose $G$ has density $g$ with respect to Lebesgue measure. Assume that $\dot{a}(y)=d a(y) / d y$ exists and

$$
\left|\frac{\dot{a}(t)}{g(t)}\right| \leq C_{1}<\infty, t \in(0,1]
$$

and

$$
\left|\frac{d(\dot{a}(t) / g(t))}{d F_{0}(t)}\right| \leq C_{2}<\infty, t \in(0,1]
$$

Then

$$
\hat{\theta}_{n}-\theta_{0}=\int b_{F_{0}} d\left(P_{n}-P\right)+o_{\mathbf{P}}\left(n^{-1 / 2}\right),
$$

where

$$
b_{F_{0}}(t, \beta)=-\beta \frac{\left(1-F_{0}(t)\right) \dot{a}(t)}{g(t)}+(1-\beta) \frac{F_{0}(t) \dot{a}(t)}{g(t)}, t \in(0,1], \beta \in\{0,1\} .
$$

Proof Condition 1 is met: $d\left(\hat{F}_{n}, F_{0}\right) \rightarrow 0$ almost surely, $\left|\hat{\theta}_{n}-\theta_{0}\right| \rightarrow 0$ almost surely and

$$
\int \log \left(\frac{p_{\hat{F}_{n}}}{p_{F_{0}}}\right) d P_{n}=O_{\mathbf{P}}\left(n^{-2 / 3}\right) .
$$

This follows from the theory in Subsection 3.1 (see also Groeneboom and Wellner (1992) and Van de Geer (1993a)). Define

$$
b_{F_{\alpha}}(t, \beta)=-\beta \frac{\left(1-F_{\alpha}(t)\right) \dot{a}(t)}{g(t)}+(1-\beta) \frac{F_{\alpha}(t) \dot{a}(t)}{g(t)}, t \in(0,1], \beta \in\{0,1\} .
$$

Then

$$
\begin{gathered}
A^{*} b_{F_{\alpha}}(y)=-\int_{y-}^{1}\left(1-F_{\alpha}(t)\right) \dot{a}(t) d t+\int_{0}^{y-} F_{\alpha}(t) \dot{a}(t) d t \\
=a(y)-a(0)-\int_{0}^{1}\left(1-F_{\alpha}(t-)\right) \dot{a}(t) d t=\psi_{F_{\alpha}}(y), y \in(0,1] .
\end{gathered}
$$


Because $F_{\alpha}$ dominates $F_{0}, d F_{0} / d F_{\alpha}$ exists. So

$$
\begin{gathered}
h_{F_{\alpha}}(y)=-\frac{d\left(F_{\alpha}(y)\left(1-F_{\alpha}(y)\right) \dot{a}(y) / g(y)\right)}{d F_{\alpha}(y)} \\
=\frac{\left(2 F_{\alpha}(y)-1\right) \dot{a}(y)}{g(y)}+\frac{F_{\alpha}(y-)\left(1-F_{\alpha}(y-)\right)}{g^{2}(y)} \frac{d(\dot{a}(y) / g(y))}{d F_{0}(y)} \frac{d F_{0}(y)}{d F_{\alpha}(y)}
\end{gathered}
$$

exists too. Moreover, for $p_{F_{\alpha}}(t, \beta)>0$,

$$
A_{F_{\alpha}} h_{F_{\alpha}}(t, \beta)=\beta \frac{\int_{0}^{t} h_{F_{\alpha}} d F_{\alpha}}{F_{\alpha}(t)}+(1-\beta) \frac{\int_{t}^{1} h_{F_{\alpha}} d F_{\alpha}}{1-F_{\alpha}(t)}=b_{F_{\alpha}}(t, \beta) .
$$

Thus, Condition 2 is fulfilled.

Clearly,

$$
\left|h_{F_{\alpha}}(y)\right| \leq C_{1}+\frac{C_{2}}{(1-\alpha)} .
$$

Therefore, Condition 3 holds too.

Finally, we shall verify Condition 4 . Note first that

$$
\int b_{F_{0}}^{2} d P=\int \frac{F_{0}(t)\left(1-F_{0}(t)\right)(\dot{a}(t))^{2}}{g(t)} d t>0
$$

and

$$
\left|b_{F_{\alpha}}(t, \beta)\right| \leq C_{1} .
$$

To check (2.10) and (2.11), we use the theory of Subsection 3.4. We have

$$
d^{2}\left(F, F_{0}\right)=\frac{1}{2}\left(\int\left(\sqrt{F}-\sqrt{F_{0}}\right)^{2} d G+\int\left(\sqrt{1-F}-\sqrt{1-F_{0}}\right)^{2} d G\right),
$$

and

$$
\int\left(b_{F_{\alpha}}-b_{F_{0}}\right)^{2} d P=\int \frac{\left(F_{\alpha}(t)-F_{0}(t)\right)^{2}(\dot{a}(t))^{2}}{g(t)} d t \leq C_{1}^{2} d^{2}\left(F_{\alpha}, F_{0}\right) .
$$

So indeed, (3.11) is satisfied: for $0<\epsilon_{n} \rightarrow 0$,

$$
\sup _{d\left(F, F_{0}\right) \leq \epsilon_{n}} \sup _{0 \leq \alpha<1} \int\left(b_{F_{\alpha}}-b_{F_{0}}\right)^{2} d P \rightarrow 0 .
$$

Also (3.8) holds, namely for $\mathcal{G}=\left\{b_{F_{\alpha}}: d\left(F, F_{0}\right) \leq \epsilon, 0 \leq \alpha<1\right\}$, we have for some constant $A$,

$$
H_{2}^{B}(\delta, \mathcal{G}, P) \leq A \frac{1}{\delta}, \delta>0
$$

This follows from entropy calculations for monotone functions (see Birman and Solomjak (1967) and, for the extension to entropy with bracketing, Van de Geer (1991)). Therefore, (2.10) and (2.11) are fulfilled. In view of Theorem 2.1, this completes the proof. 
The result of Lemma 4.1 was established earlier in Groeneboom and Wellner (1992). They use specific properties of the maximum likelihood estimator $\hat{F}_{n}$, such as the distance between successive jumps of $\hat{F}_{n}$ being smaller than $n^{-1 / 3} \log n$ with large probability. In this sense, local properties of $\hat{F}_{n}$ had to be obtained first, before one could arrive at the asymptotic behaviour of such global quantities as the mean of the maximum likelihood estimator. It inspired us to develop an alternative proof, which is hopefully applicable in more general situations.

The model for interval censored observations gives a good insight into the difficulties that arise due to the fact that $\hat{F}_{n}$ does not dominate $F_{0}$. Let us have a closer look for the case $a(y)=y$. It is known that $\hat{F}_{n}$ has finite support, say $\hat{z}_{1}<\ldots<\hat{z}_{m}$. Define

$$
\hat{g}(t)=\frac{G\left(\hat{z}_{j}\right)-G\left(\hat{z}_{j-1}\right)}{\hat{z}_{j}-\hat{z}_{j-1}}, t \in\left(\hat{z}_{j-1}, \hat{z}_{j}\right],
$$

and

$$
b_{\hat{F}_{n}}(t, \beta)=-\beta \frac{1-\hat{F}_{n}(t)}{\hat{g}(t)}+\beta \frac{\hat{F}_{n}(t)}{\hat{g}(t)} .
$$

Then for $y \in\left\{\hat{z}_{1}, \ldots, \hat{z}_{m}\right\}$,

$$
A^{*} b_{\hat{F}_{n}}(y)=y-\hat{\theta}_{n}=\psi_{\hat{F}_{n}}(y)
$$

However,

$$
\int \psi_{\hat{F}_{n}} d F_{0}=-\left(\hat{\theta}_{n}-\tilde{\theta}_{n}\right)
$$

with

$$
\tilde{\theta}_{n}=\iint^{y} \frac{1}{\hat{g}(t)} d G(t) d F_{0}(y) .
$$

In general, $\tilde{\theta}_{n} \neq \theta_{0}$, unless $G$ happens to be the uniform distribution on $(0,1]$.

Write

$$
\begin{gathered}
h_{\hat{F}_{n}}(y)=-\frac{d\left(\hat{F}_{n}(y)\left(1-\hat{F}_{n}(y)\right) / \hat{g}(y)\right)}{d \hat{F}_{n}(y)} \\
=\frac{2 \hat{F}_{n}(y)-1}{\hat{g}(y)}+\frac{\hat{F}_{n}(y-)\left(1-\hat{F}_{n}(y-)\right)}{\hat{g}(y) \hat{g}(y-)} \frac{d \hat{g}(y)}{d \hat{F}_{n}(y)} .
\end{gathered}
$$

In order to have that at $\hat{F}_{n}$, the derivative of the likelihood equals zero in the direction $h_{\hat{F}_{n}}$, one must have that this direction is bounded. This leads to showing that the jumps of $\hat{F}_{n}$ are large enough.

\section{EXample 4.2: interval CEnsored observations: CASE II}

Let $X_{i}=\left(T_{i}, U_{i}, \beta_{i}, \gamma_{i}\right)$, with $\beta_{i}=1\left\{Y_{i} \leq T_{i}\right\}, \gamma_{i}=1\left\{T_{i}<Y_{i} \leq U_{i}\right\}$ and $Y_{i}$ independent of $\left(T_{i}, U_{i}\right), i=1, \ldots, n$. We assume bounded support, say $T_{i}, U_{i}$ and $Y_{i} \in(0,2]$, and that $T_{i}<U_{i}$. This model is also studied in Groeneboom and Wellner (1992). The rate of convergence of the log-likelihood ratio can 
be found in Van de Geer (1993b). In general, no explicit expression for the influence curve can be given. However, it can be shown that under fairly mild conditions, $b_{\hat{F}_{n}}$ and $h_{\hat{F}_{n}}$ exist and are bounded. One of the conditions here is that $T_{i}$ and $U_{i}$ can be arbitrary close to each other. We shall now consider a situation where this is not true, but where explicit expressions are available. Namely, we suppose that $U_{i}=T_{i}+1$. Let $G\left(F_{0}\right)$ be the distribution of $T_{i}\left(Y_{i}\right)$, with density $g\left(f_{0}\right)$ w.r.t. Lebesgue measure.

Suppose that

$$
\begin{aligned}
& 0<1 / C_{0} \leq f_{0}(y) \leq C_{0}<\infty, y \in(0,2], \\
& \frac{|\dot{a}(t+1)| \vee|\dot{a}(t)|}{g(t)} \leq C_{1}<\infty, t \in(0,1]
\end{aligned}
$$

and

$$
\left|\frac{d(\dot{a}(t+1) / g(t))}{d F_{0}(t+1)}\right| \vee\left|\frac{d(\dot{a}(t) / g(t))}{d F_{0}(t)}\right| \leq C_{2}<\infty, t \in(0,1] .
$$

Then

$$
\begin{aligned}
b_{F_{0}}(t, \beta, \gamma) & =\beta \frac{H_{F_{0}}(t)}{F_{0}(t)}+\gamma \frac{H_{F_{0}}(t+1)-H_{F_{0}}(t)}{F_{0}(t+1)-F_{0}(t)} \\
& -(1-\beta-\gamma) \frac{H_{F_{0}(t+1)}}{1-F_{0}(t+1)},
\end{aligned}
$$

and

$$
H_{F_{0}}(y)= \begin{cases}F_{0}(y)\left(F_{0}(y)+F_{0}(y+1)-2\right) \frac{\dot{a}(y)}{g(y)}, & 0<y \leq 1, \\ \left(F_{0}(y-1)+F_{0}(y)\right)\left(1-F_{0}(y)\right) \frac{\dot{a}(y)}{g(y-1)}, & 1<y \leq 2\end{cases}
$$

The directions are $h_{F_{\alpha}}=d H_{F_{\alpha}} / d F_{\alpha}$, with $H_{F_{\alpha}}$ of the form (4.7), with $F_{0}$ replaced by $F_{\alpha}$. However, condition 3 is not met, because $d F(y+$ 1) $/ d F_{0}(y)$ and $d F(y) / d F_{0}(y+1), 0<y \leq 1$, can be arbitrary large. It is not clear to us whether the maximum likelihood estimator will be efficient. So this example shows that Theorem 2.1 certainly does not always provide an answer.

\section{EXAMPLE 4.3: CONVOLUTION.}

Let $X_{i}=Y_{i}+Z_{i}$, with $Y_{i}$ and $Z_{i}$ independent, $Y_{i}$ has unknown distribution $F_{0}$ and $Z_{i}$ has known distribution $K, i=1, \ldots, n$. Suppose $K\left(F_{0}\right)$ has density $k\left(f_{0}\right)$ w.r.t. Lebesgue measure and that $K$ and $F_{0}$ have support in $(0,1]$. We consider the special case

$$
k(z)=c_{1}^{z} / c_{2}, 0<z \leq 1, c_{2}=\frac{\log c_{1}}{c_{1}-1}
$$

where $c_{1} \geq 1$ is fixed.

Lemma 4.3. Suppose

$$
0<1 / C_{0} \leq f_{0}(y) \leq C_{0}<\infty, 0<y \leq 1,
$$


and

$$
|d \dot{a}(y) / d y| \leq C_{1}<\infty
$$

Then

$$
\hat{\theta}_{n}-\theta_{0}=\int b_{F_{0}} d\left(P_{n}-P\right)+o_{\mathbf{P}}\left(n^{-1 / 2}\right),
$$

where

$$
\begin{gathered}
b_{F_{0}}(x)= \begin{cases}\frac{\Phi_{F_{0}}(x)}{L_{F_{0}}(x)}, & 0<x \leq 1 \\
\frac{\Phi_{F_{0}}(1)-\Phi_{F_{0}}(x-1)}{L_{F_{0}}(1)-L_{F_{0}}(x-1)}, & 1<x \leq 2\end{cases} \\
\Phi_{F_{0}}(y)=\frac{c_{1} L_{F_{0}}(y) \Phi_{F_{0}}(1)-\left(\dot{a}(y)+\log c_{1}\left(a(y)-\theta_{0}\right)\right) c_{2} L_{F_{0}}(y)\left(L_{F_{0}}(1)-L_{F_{0}}(y)\right)}{L_{F_{0}}(1)+\left(c_{1}-1\right) L_{F_{0}}(y)}
\end{gathered}
$$

and

$$
L_{F_{0}}(y)=\int_{0}^{y} c_{1}^{-u} d F_{0}(u) .
$$

Proof Let us only check (2.4) of Condition 1 . The other conditions can be verified in the same way as in Lemma 4.1. If $c_{1}=1$, then (2.4) also follows from the same arguments as in Lemma 4.1, and we find

$$
\int \log \frac{p_{\hat{F}_{n}}}{p_{F_{0}}} d P_{n}=O_{\mathbf{P}}\left(n^{-2 / 3}\right) .
$$

For $c_{1}>1$, the class $\mathcal{K}=\{k(\cdot, y)=k(\cdot-y), y \in(0,1]\}$ satisfies for some $A$,

$$
N(\delta, \mathcal{K}, Q) \leq A \delta^{-1}
$$

for all $\delta>0$ and all probability measures $Q$. Moreover, $\mathcal{K}$ is uniformly bounded. Now,

$$
P_{F_{0}}(x)= \begin{cases}\frac{c_{1}^{x}}{c_{2}} \int_{0}^{x} c_{1}^{-y} d F_{0}(y), & 0<x \leq 1, \\ \frac{c_{1}^{x}}{c_{2}} \int_{x-1}^{1} c_{1}^{-y} d F_{0}(y), & 1<x \leq 2\end{cases}
$$

Using (4.8), one sees that in Lemma 3.2, one can take $\tau_{1}^{2}(\sigma) \asymp \sigma$ and $\tau_{2}^{2}(\sigma) \asymp$ $\log (1 / \sigma)$ for $\sigma>0$ small. So, inserting $w=1$ in this lemma, one obtains

$$
\int \log \left(\frac{2 p_{\hat{F}_{n}}}{p_{\hat{F}_{n}}+p_{F_{0}}}\right) d P_{n}=O_{\mathbf{P}}\left(n^{-3 / 4}(\log n)^{1 / 4}\right) .
$$

\section{REFERENCES}

Birgé, L. and Massart, P., (1993), Rates of convergence for minimum contrast estimators, Probab. Th. Relat. Fields 97 113-150.

Birman, M. and Solom.jak, M.J., (1967), Piece-wise polynomial approximations of functions of the classes $W_{p}^{\alpha}$, Mat. Sbornik 73 295-317.

Groeneboom, P. and Wellner, J.A., (1992), Information Bounds and Nonparametric Maximum Likelihood Estimation, BMW Seminar, Band 19, Birkhäuser. 
OssiAnDER, M. (1987), A central limit theorem under metric entropy with $L_{2}$ bracketing, Ann. Probab. 15 897-919.

Pfanzagl, J., (1988), Consistency of maximum likelihood estimators for certain nonparametric families, in particular: mixtures, J. Statist. Plann. Inference 19 137-158.

Pollard, D. (1984), Convergence of Stochastic Processes, Springer, New York.

VAN DE GEER, S. (1990), Estimating a regression function, Ann. Statist. 18 907-924.

VAN DE GEER, S. (1991), The entropy bound for monotone functions, Techn. Report TW 91-10, Univ. of Leiden.

VAN DE GEER, S. (1993a), Hellinger consistency of certain nonparametric maximum likelihood estimators, Ann. Statist. 21 14-44.

VAN DE GEER, S. (1993b), Rates of convergence for the maximum likelihood estimator in mixture models, Techn. Report TW 93-09, Univ. of Leiden.

VAN DE GEER, S. (1995), The method of sieves and minimum contrast estimators, Mathematical Methods of Statistics 4 20-38.

VAN DER LAAN, M.J. (1993), Efficient and Inefficient Estimation in Semiparametric Models Thesis, Univ. of Utrecht. To appear as CWI tract 44, Centre for Math. and Comp. Sci., Amsterdam.

VAN Der LAan, M.J. (1994), Proving efficiency of NPMLE and identities, Techn. Report 44, Un. of California, Berkeley.

VAN DER VAART, A.W. (1991), On differentiable functionals, Ann. Statist. 19 178-205.

Wong, W.H. and SHEN, X. (1992), Probability inequalities for likelihood ratios and convergence rates for sieve MLE's, Techn. Report 346, Univ. of Chicago.

Mathematics Department, Leiden University, Netherlands 